\title{
System Design of a Hand-Held Mobile Robot for Craniotomy
}

\author{
Gavin Kane ${ }^{1}$, Georg Eggers ${ }^{1}$, Robert Boesecke ${ }^{1}$, Jörg Raczkowsky ${ }^{2}$, Heinz Wörn ${ }^{2}$, \\ Rüdiger Marmulla ${ }^{1}$, and Joachim Mühling ${ }^{1}$ \\ ${ }^{1}$ Klinik und Poliklinik für Mund-, Kiefer, und Gesichtschirurgie, University of Heidelberg, \\ Heidelberg, Germany \\ ${ }^{2}$ Institute for Process Control and Robotics, University of Karlsruhe, Karlsruhe, Germany
}

\begin{abstract}
This contribution reports the development and initial testing of a Mobile Robot System for Surgical Craniotomy, the Craniostar. A kinematic system based on a unicycle robot is analysed to provide local positioning through two spiked wheels gripping directly onto a patients skull. A control system based on a shared control system between both the Surgeon and Robot is employed in a hand-held design that is tested initially on plastic phantom and swine skulls. Results indicate that the system has substantially lower risk than present robotically assisted craniotomies, and despite being a hand-held mobile robot, the Craniostar is still capable of sub-millimetre accuracy in tracking along a trajectory and thus achieving an accurate transfer of pre-surgical plan to the operating room procedure, without the large impact of current medical robots based on modified industrial robots.
\end{abstract}

\section{Introduction}

There are a number of surgical craniotomy procedures in which it is desired for a precise pre-surgical plan to be accurately transferred to the Operating Room (OR), such procedural examples include minimally invasive neuro-surgical procedures where the desired entry hole is pre-planned, frontal orbital advancement procedures for maxillofacial surgery where the desired cranium advancement is pre-planned, or in a more recent example the milling of bone surfaces in plastic surgery according to a three dimensional operation plan [1] or CAD/CAM prefabricated skull implants requiring accurate bone resection for placement [2]. In support of this requirement for accurate transfer, many research groups have developed robotic solutions to address the specific challenges associated with the conduct of a craniotomy procedure [2], [3], [4]. To date, none of these solutions have been accepted for commercial use in performing craniotomys, it is assessed due to three facts:

- The surgical robotic systems presented are modified industrial robots whose impact within the OR in terms of footprint and the required changes to surgical workflows and procedures is quite considerable.

- The risk inherent with the demonstrated robotic craniotomys are considerably large, with respect to cutting too deep, causing meninges tears, and possibly thereafter damage to the brain. 
- The robotic solutions offered are all supervisory controlled interventions where, by the definition of Nathoo et al. [5], the robot performs the pre-planned and programmed movements autonomously, thus removing the surgeon from the procedure. This occurring at the key time when his / her years of experience and 'feel' for the operation are most valuable.

Shared control is not a new concept in Surgical Robotics. Synergistic control was proposed by Taylor et al. [6] as early as 1991. Recent examples of this control in surgical robots include the Acrobot [7] and the PADyC system [8]. However, these two systems are still modified industrial robots, whose large footprint and individualized workflows still create a substantial impact to the OR. There are also no similar systems developed for the conduct of craniotomys.

There is an alternate trend in development, which is heading towards the minimisation of the impact of robotics in the OR: through the use of intelligent hand-held tools. Example projects in this area include the Precision Freehand Sculptor (PFS) by Brisson et al. [9] and the Sicherheits Trepenation System (STS) by Follman et al [10]. The PFS demonstrates how bone segments, such as those for knee implants, can be accurately removed according to a pre-planned model. On the other hand, the STS demonstrates how a normal hand-held drill can be enhanced for safety with the addition of both intrinsic and extrinsic system sensors. Unfortunately, this second system is limited to safety enhancement, and is not capable of any assisting the Surgeon in achieving an accurate pre-planned trajectory in line with the previously stated requirements.

From all the above examples, there is still no developed capability for a safe shared control system for craniotomys, specifically capable of accurately milling pre-planned trajectory in the OR with minimal impact on OR footprint and workflows. The solution presented here is a novel approach to Surgical Robotics, which addresses the following hypothesis:

1. A mobile robot, built around a standard craniotomy drill piece, hand-held by the surgeon, can drive the drill piece around a skull, on a pre-defined trajectory.

2. The system shall be capable of achieving surgical accuracy with the use of spiked wheels to ensure the skull remains a non-slip surface for dynamic analysis.

3. The control system supporting the robot is capable of guiding the surgeons hand during a procedure, but is not to remove him/her from complete control. i.e. This is to be a shared control system by the definition in [5].

In this paper we present the design, theory and performance testing of the prototype robot system, the 'Craniostar' shown in Fig. 1.

\section{Methods and Materials}

One of the concepts for using a mobile hand-held robot discussed above was the minimisation of the impact into the Operating Room. The components accepted as standard for Image Guided Surgery (IGS) include a tracking system and imaging support (GUI). All attempts were then made to minimise additional footprint requirements (that of the robot and robot control) but maximise the integration of the system into the existing OR. A key component of this integration was the generation of an 


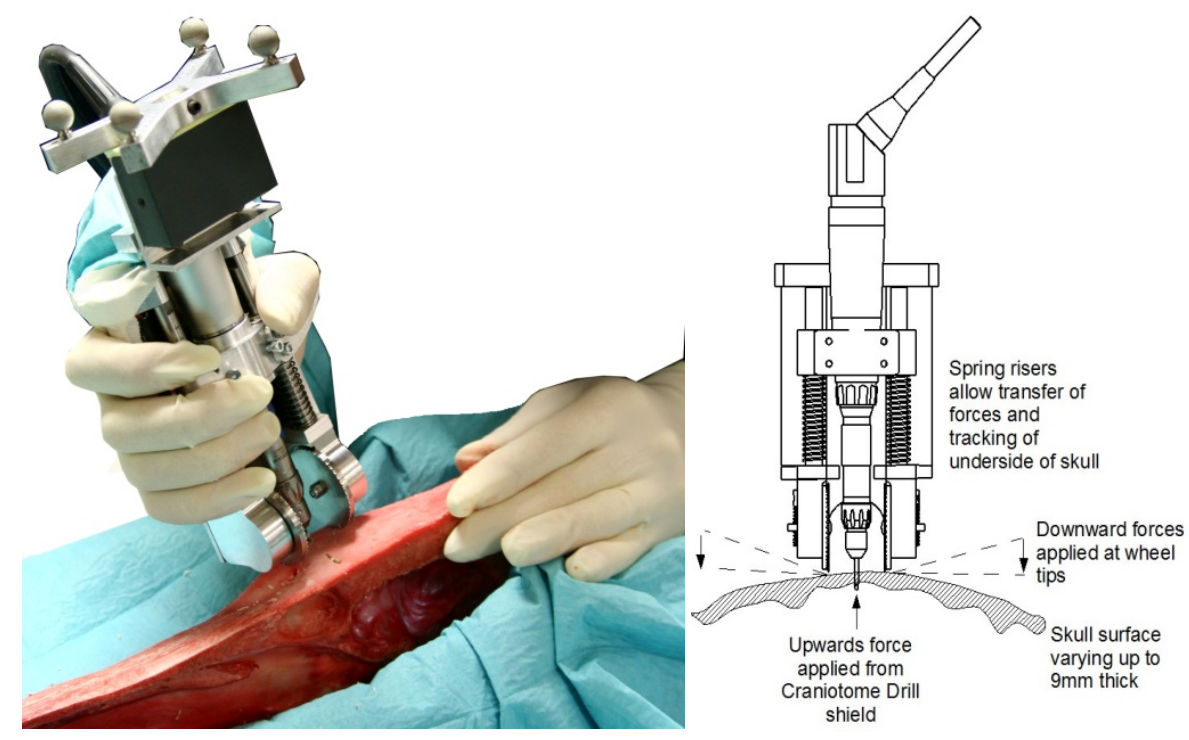

Fig. 1. (a) The prototype mobile hand-held surgical robot, 'Craniostar' being tested on a cadaver, and (b) a CAD drawing showing the principle forces used to ensure traction

intuitive Human / Computer Interface (HCI). The HCI is based on employing three inputs for the robot, from components already existing inside the OR. The first is the use of the Navigation System. The navigation system detecting minor tilts of the robot, occurring when the Surgeon pushes the robot forward implying an intent to move faster, or pulling the robot backwards implying an intent to slow down or stop. The second and third inputs are from the existing high speed drill, the foot pedal input for the desired speed of the drill, and the torque feedback of the drill for the difficulty the robot is facing in cutting. By integrating this control into the system, [11] showed that a minimal impact, intuitive shared control could be achieved.

The overall concept for employment follows a generalised IGS workflow with commencement of pre-surgical imagery and planning. Here a CT is employed to support the surgeons planning the Craniotomy through the KASOP software[12]. The location for the start of the Craniotomy is determined using the GUI and either an optically tracked pointer or the tip of the Craniostar itself, and the first burr hole is made. If required the Dura matter can be partially separated from the underside of the skull. If a circular craniotomy is desired, no further burr holes are required, and the tip of the Craniostar is inserted facing roughly in the correct direction. For a two ended craniotomy, a second bore hole is required at the finish location. The control of the movement of the Craniostar along the trajectory is discussed in the following sections on steering and control.

Firstly, this chapter will discuss how this system was achieved through a custom mechanical design, and then the control system is discussed. 


\subsection{Mechanical Design}

The concept for the mobile robot, shown in Fig. 1, comprises a platform (robot body), two wheels, and the craniotomy high speed drill directly at the centre of the wheels' axis, with an optical tracking marker placed on the top of the robot. The high speed drill (Aesculap AG, Tuttlingen, Germany) is held in a brace in the centre of the platform. The brace rests on two spring supported sliders, allowing the highspeed drill to move up and down tracking the base of the skull. The spring force generated on the sliders has two purposes, firstly to prevent the drill and Dura Guard being pushed hard against the Dura, and secondly in order to transfer force to the wheels of the robot, supporting the friction of the wheels and hence the traction. The required spring strength was calculated based on the ability of the Dura Guard to track the bottom of the skull, with depth changes to a maximum of $9 \mathrm{~mm}$ while moving up to a maximum cutting speed of $5 \mathrm{~mm} / \mathrm{s}$. The Dura Guard being a small metal hook that extends underneath the drill piece, preventing the drill from cutting into the Dura.

The motor drive is achieved through two 25W Motors (Maxon Motors GmBH, Sexau, Germany) with embedded position encoders providing velocity and position feedback. To ensure the drive is capable of sub-millimeter positioning accuracy, the drive was engineered with two gearing stages. Firstly internal to the motors are 19:1 planetary gearboxes, with an average backlash under no load of $1.2^{\circ}$. A second stage involves a worm wheel drive to an anti-backlash gear with a 60:1 reduction. With this final gear connected directly to the wheel $(\varnothing 40 \mathrm{~mm})$, the final play in the system equates to a theoretical positioning ability of $0.006 \mathrm{~mm}$. Secondly, with a wheel axle separation of $24 \mathrm{~mm}$, a theoretical forward angle alignment of $0.014^{\circ}$ is achieved.

Unfortunately greater inaccuracies in the system lie in two fundamental areas, the ability of the wheels to maintain traction on the skull, and in the position feedback of the robot and patient tracking. The second inaccuracy relies on the Polaris Optical Tracking System (Northern Digital Inc., Ontario, Canada), which has known inaccuracies of $0.4 \mathrm{~mm}$. The first inaccuracy the traction; however, is more complex and the larger unknown to the system.

The ability of the robot to maintain traction on the skull is unfortunately open to a great deal of unknowns, the skulls curvature or anomalies or contaminates (eg. water, blood or other fluids). Prior to modeling these factors, it was decided to make an overall conceptual test of the friction available from two possible wheel models, shown in Fig. 2: design 1 with an ability to penetrate through any possible surface contaminates, and design 2 for possibly softer, thinner bone (eg children) with a safety flange to prevent complete penetration should too much pressure be placed on the wheels. The result of the testing is discussed in the next section.

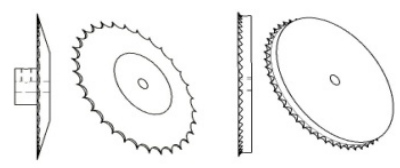

Fig. 2. Two separate wheel designs were prepared for testing on a Swine skull. Design 1 (left) had $2 \mathrm{~mm}$ spikes, Design 2 (right) had $1 \mathrm{~mm}$ spikes, with a safety flange to prevent excessive penetration. 


\subsection{Steering Control System}

The required control system here appears similar to that of the collaborative robots (COBOTs) in the work by Peshkin et al. [13]. However, Peshkin defines COBOTs as intrinsically passive devices using "steerable" non-holonomic joints. Instead the Craniostar was designed with driven wheels, providing steering and additionally a greater level of support with the surgeon's choice of speed, assisting in the forward movement of the device within a set tilt limitation. This tilt limitation also ensures that the cutting tool is used only when very close to vertical; these capabilities would not have been possible through the application of the COBOT control methods. Instead dynamic path following control methods were analysed from a number of papers e.g. [[14],[15],[16]]. The choice was made to test the control algorithm proposed by Seo $\mathrm{K}$. et al [15] for a number of reasons.

- Firstly, the approach does not assume perfect system performance and is designed to cope with uncertainty in dynamics arising from path following.

- Secondly, the approach is not restricted to constant path curvature.

- Thirdly, the algorithm provides the mechanism for definition of a safety boundary. In the initial definition, this safety boundary is an area where the mobile robot may move free from collisions; however, when employing the algorithm in a medical application, the safety boundary is redefined as the area within which it is 'safe' to operate.

A lot of research into unicycle kinematics of mobile robots, is based on the movement of the wheels over a flat surface defined in terms of $\mathrm{x}$ and $\mathrm{y}$ coordinates, for example see [[14]-[16]]. Fig. 3 (left) shows some of the standard parameters for a unicycle robot. Unfortunately this simplistic $2 \mathrm{D}$ view of the surface is not perfectly compatible with highly curved and irregular surfaces (eg a skull). An initial experimentation with translation to spherical coordinates was made on a standard model phantom skull. It was determined that the simplistic solution was feasible; however, on complexities that arise out of abnormal skull shapes, such as those found in surgeries requiring birth defect or accident trauma cranial reconstruction, this did not work.
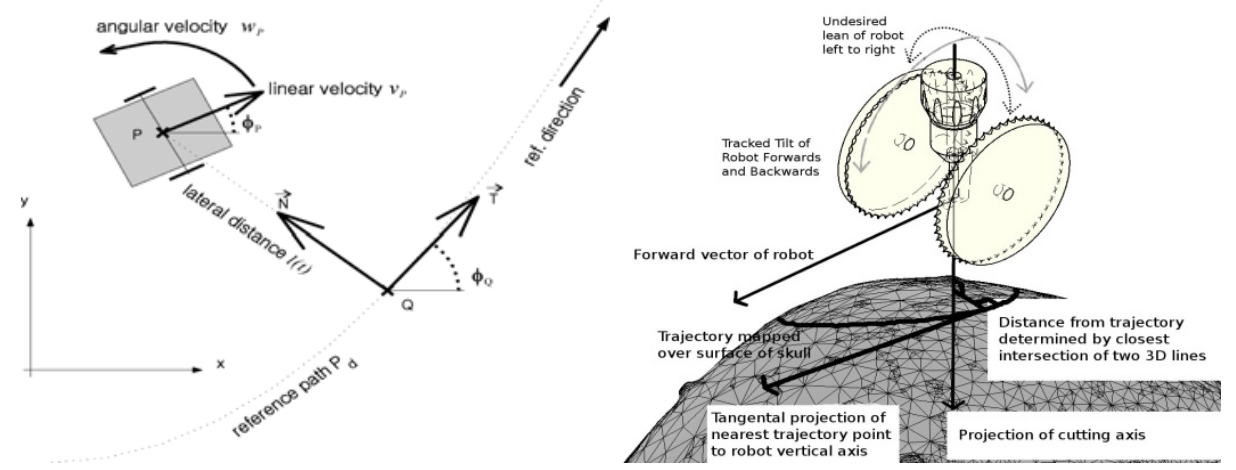

Fig. 3. Original 2D parameters (left)(Duplicated with permission from Seo K.) and the 3D derivation (right) of the parameters for the Craniostar involving the projection of virtual vectors through the cutting axis of the robot and the closest trajectory segment to this vector. 
Instead, we used an approach that entirely ignored the surface. All control parameters were extracted from abstract virtual 3D vectors based on the position of the robot, and the trajectory. Equations to determine the control parameters, discounted the actual intersection with the skull, and for example, looked more directly at the 3D closest segment between two lines such as the closest trajectory segment, and a virtual projection through the drilling axis. The trajectory is known to lie on the skull, and it is known the robot operates with both wheels on the skull, therefore, it can be assumed that the closest line between these two aforementioned vectors will closely approximate the lateral distance on the skull. Fig. 3 displays some of these abstractions. These parameters were then employed in accordance with [15], the key equations being duplicated here: the steering controller in Equation 1, and the sliding mode between safety regions defined in Equation 2. The parameters are defined as per Fig 3, with tuneable parameters $\mathrm{k}, \mathrm{b}_{1}$, and $\epsilon$ defining the path approach.

$$
\begin{gathered}
w^{*}\left(s(t), l(t), \tilde{\phi}(t), v_{p}(t)\right)=\frac{\kappa(s(t)) v_{p}(t) \cos (\tilde{\phi}(t))}{1-\kappa(s(t)) l(t)}-\frac{k v_{p}(t) \sin (\tilde{\phi}(t))}{1+(k l(t))^{2}}-\left(\omega_{p}+b_{1}\right) \operatorname{sat}\left(\frac{z(t)}{\epsilon}\right) . \\
z(t)=\tilde{\phi}(t)+\arctan (k l(t))-2 \pi m, m \in \mathbb{R} .
\end{gathered}
$$

\section{Results}

The testing of the Craniostar robot to meet the system requirements, in terms of accuracy, feasibility and safety has commenced in two separate phases.

- The accuracy of the control system was tested initially on flat surfaces and then on phantom skulls, and

- The feasibility and safety aspects were tested under OR conditions on Swine skulls. See Fig. 1.

The tests on the accuracy of the control system are outlined in table 1. Two known inaccuracies lead to less than $100 \%$ of the accuracies under the $\pm 0.5 \mathrm{~mm}$ region. The first in the initial placement of the robot, while the software's visual GUI can guide the surgeon in its placement, it is still found quite difficult to place the robot truly precisely. The recovery from this initial placement occurs quickly, within approximately $2 \mathrm{~mm}$ of travel. But it is accepted that the initial hole size for inserting the Craniotomy Tool Piece with Dura Guard is larger than this inaccuracy, and is therefore deemed negligible. This is seen in Fig. 4(a) where an initial placement offset was quickly recovered.

Table 1. Average accuracies achieved by the Craniostar on different trajectories

\begin{tabular}{llcc}
\hline & & \multicolumn{2}{c}{ Average accuracy achieved } \\
\hline Surface & Trajectory & $\pm 0.5 \mathrm{~mm}$ & $\pm 1 \mathrm{~mm}$ \\
\hline Flat Wood & Single straight $5 \mathrm{~cm}$ segment & $97.20 \%$ & $100.00 \%$ \\
& Single curved $90^{\circ}$ segment with 4cm radius & $98.15 \%$ & $100.00 \%$ \\
& Two 5cm segments joined with 45 join & $97.60 \%$ & $100.00 \%$ \\
\hline Plastic & Single straight 5cm segment & $97.00 \%$ & $100.00 \%$ \\
Phantom & Two 5cm segments joined with 45 join & $95.00 \%$ & $100.00 \%$ \\
Skull & Single curved $90^{\circ}$ segment with 4cm radius & $95.60 \%$ & $100.00 \%$ \\
\hline
\end{tabular}



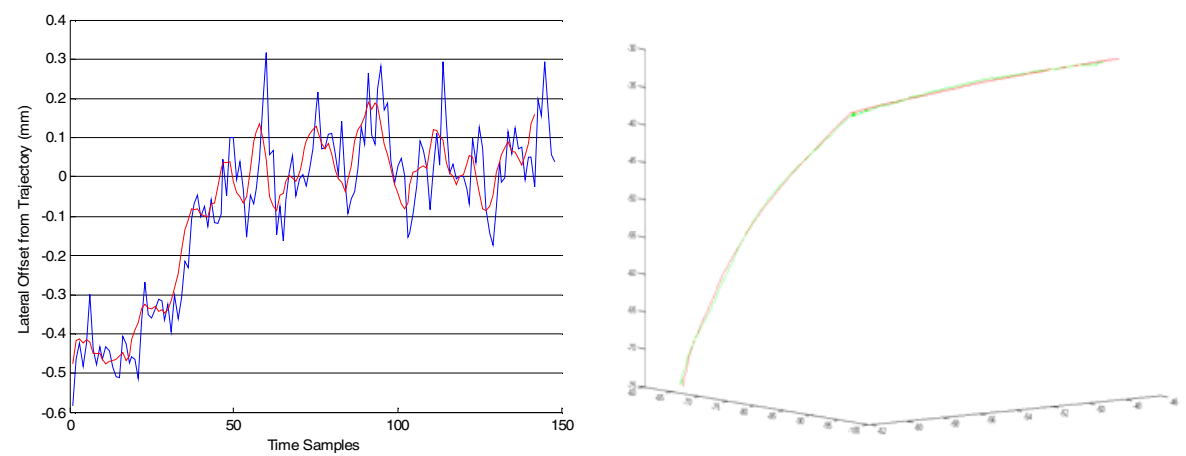

Fig. 4. Tracking examples. (a) The lateral distance (error) of a $5 \mathrm{~cm}$ straight track showing an initial placement error of the Craniostar, and the following robots correction. Noise in graph is primarily due to Optical Tracking. (b) The 3D Track plot of two joined segments, curving over the surface of the phantom skull (red line, planned trajectory, green line tracked, distances $\mathrm{mm}$ )

The second error lies in the nature of the shared control of the system. The hand-held device is by nature prone to noise, specifically in external forces from the surgeons hand. Fig. 4(b) show a trajectory being tracked by a Surgeon over a skull. The intersection between the two segments has a slight disturbance in the middle due to the requirement for sharp turning at the segment intersection. In order to achieve the desired $\pm 0.5 \mathrm{~mm}$ here, took a degree of education for the user into knowing what the robot is likely to do, this

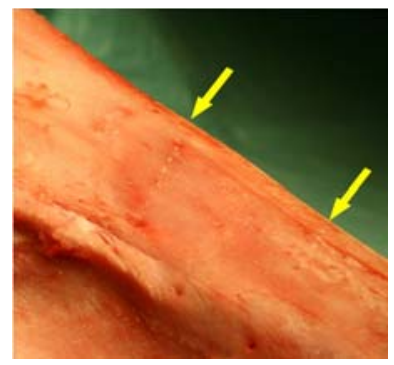

Fig. 5. The superficial marks of the Craniostars motion over the swines skull required enhancements to the Graphical User Interface, feeding back to the Surgeon to give advanced warning of such likely intent.

The testing on a Swine skull in phase two involved the impact on the skull and frictional testing. The results of this testing demonstrated no ability of the spiked wheels to damage the skull, though superficial tracks did remain on the skull immediately following the robots movements, visible in Fig. 5. The testing also confirmed the robot's ability to move over the skull without any wheel slippage, thus supporting the choice of control theory with non-sliding kinematics.

\section{Conclusion}

In this paper, a new concept of surgical robotics was proposed and developed using a hand-held mobile robot to aid the surgeon in the conduct of a craniotomy. It shows the possibility for achieving precision pre-surgical planning to OR transfer, without removing the Surgeon from the procedure, and without a large impact on OR footprint and surgical workflows. Future work still includes minaturisation of the gearing section and development of a more precise friction model of the skull for refining the control system prior to moving towards clinical trials. 
Acknowledgment. This EU project "CompuSurge" is funded by the "Marie Curie" research network. A collaboration between the University Hospital Heidelberg and the Institute of Process Control and Robotics at Karlsruhe University.

\section{References}

1. Hassfeld, S., Muehling, J.: Computer assisted oral and maxillofacial surgery-a review and an assessment of technology. Int. J. Oral Maxillofac. Surg. 30, 2-13 (2001)

2. Weihe, S., Schiller, C., Rasche, C., Hassfeld, S., Wehmöller, M., Knoop, H., Epple, M., Eufinger, H.: CAD-CAM prefabricated individual skull implants: new aspects in robot resection and guided bone regeneration. International Congress Series 1268, 584-590 (2004)

3. Engel, D., Raczkowsky, J., Wörn, H.: RobaCKa- Ein Robotersystem für den Einsatz in der Chirurgie. In: Rechner- und sensorgestützte Chirurgie, Proceedings zum Workshop, vol. 4, pp. 279-286 (2001)

4. Bast, P., Popovic, A., Wu, T., Heger, S., Engelhardt, M., Lauer, W., Radermacher, K., Schmeider, K.: Robot- and computer-assisted craniotomy: resection planning, implant modelling and robot safety. Int. J. Med. Robotics. Comput. Assist. Surg. 2, 168-178 (2006)

5. Nathoo, N., Cavusoglu, M., Vogelbaum, M.A.: In touch with robotics: neurosurgery for the future. Neurosurgery 56, 421-433 (2005)

6. Taylor, R.H., et al.: A model-based optimal planning and execution system with active sensing and passive manipulation for augmentation of human precision in computer-integrated surgery. In: 2nd International Conference on Experimental Robotics, Toulouse, France (1991)

7. Matjaz, J., Harris, S., Baena, F.R., Gomes, P., Davies, B.L.: The Acrobot system for total knee replacement. Industrial Robot: An International Journal 30, 61-66 (2003)

8. Schneider, O., Troccaz, J.: A Six-Degree-of-Freedom Passive Arm with Dynamic Constraints (PADyC) for Cardiac Surgery Application: Preliminary Experiments. Comp. Aid. Surg. 6, 340-351 (2001)

9. Brisson, G., Kanade, T., DiGioia, A., Jaramaz, B.: Precision Freehand Sculpting of Bone. In: Barillot, C., Haynor, D.R., Hellier, P. (eds.) MICCAI 2004. LNCS, vol. 3217, pp. 105 112. Springer, Heidelberg (2004)

10. Follman, A., Little, J., Schröder, K., Forff, A., Engelhardt, M., Radermacher, K.: Synergistic Control - Semiautomatiche Navigation eines neuartigen Trepanationsinstrumentes. In: CURAC 2008, pp. 111-112 (2008)

11. Kane, G., Eggers, G., Boesecke, R., Raczkowsky, J., Wörn, H., Marmulla, R., Mühling, J.: Intuitively Controlled Handheld Mobile Robot for Precision Craniotomy Surgery. In: 13th International Conference on Human-Computer Interaction (in print, 2009)

12. Schorr, O., Münchenberg, J., Raczkowsky, J., Wörn, H.: KasOp - A Generic System for Pre- and Intraoperative Surgical Assistance and Guidance. In: 15th International Congress and Exhibition of Computer Assisted Radiology and Surgery, CARS (2001)

13. Wannasuphoprasit, W., Gillespie, R.B., Colgate, J.E., Peshkin, M.A.: Cobot Control. In: International Conference on Robotics and Automation (1997)

14. Samson, C.: Path following and time-varying feedback stabilization of a wheeled mobile robot. In: International Conference on Advanced Robotics and Computer Vision, vol. 13, pp. 1-5 (1992)

15. Seo, K., Lee, J.S.: Kinematic path-following control of a mobile robot under bounded angular velocity error. Advanced Robotics 20, 1-23 (2005)

16. Kim, B., Tsiotras, P.: Controllers for Unicycle-Type Wheeled Robots: Theoretical Results and Experimental Validation. IEEE Transactions on Robotics and Automation 18, 294 307 (2002) 